\title{
Fatores associados à coinfecção Leishmania/Vírus da Imunodeficiência Humana: um estudo de coorte (2007-2018)
}

Factors associated with Leishmania/Human Immunodeficiency Virus co-infection: a cohort study (2007-2018)

Factores asociados con la coinfección por Leishmania / Virus de la Inmunodeficiencia Humana: un estudio de cohorte (2007-2018)

\section{Resumo}

Introdução: Leishmaniose visceral (LV) e infecção pelo vírus da imunodeficiência humana (HIV) são problemas de saúde pública, com altos números de casos e letalidade no mundo. Objetivo: Avaliar os fatores associados a coinfecção leishmania/HIV no Brasil. Metodologia: Estudo de coorte retrospectiva com análise univariada e multivariada de 28.265 indivíduos, notificados e com diagnóstico confirmado de LV, no Sistema Nacional de Agravos de Notificação (SINAN), de 2007 a 2018, no Brasil. Resultados: A maioria dos indivíduos coinfectados era jovem, homem, da raça não branca, moradores da zona urbana, com até oito anos de escolaridade e que entraram nesse estudo como caso novo. Maiores chances de coinfecção foram observadas para indivíduos de faixas etárias de onze a dezenove anos $(\mathrm{OR}=1,74)$, vinte a trinta e nove anos $(\mathrm{OR}=13,06)$ e acima de quarenta anos $(\mathrm{OR}=6,96)$, que fizeram uso de antimoniato de $\mathrm{N}$-metilglucamina $(\mathrm{OR}=4,36)$, anfotericina $\mathrm{B}$ desoxicolato $(\mathrm{OR}=6,21)$ e anfotericina $\mathrm{B}$ lipossomal $(\mathrm{OR}=1,60)$, que apresentaram recidiva $(\mathrm{OR}=3,99)$, cujos casos evoluíram para abandono de tratamento $(\mathrm{OR}=2,00)$, e óbitos por outras causas que não LV $(\mathrm{OR}=4,00)$ e transferência $(\mathrm{OR}=1,50)$. Menores chances de coinfecção foram observadas em mulheres $(\mathrm{OR}=0,80)$, moradores da zona rural $(\mathrm{OR}=0,54)$, que apresentaram de cinco a seis sintomas $(\mathrm{OR}=0,82)$. Conclusão: $\mathrm{Os}$ resultados apontam para a necessidade de melhoria no acompanhamento de indivíduos coinfectados com LV/HIV, no intuito de diminuir recidivas, transferências e abandono ao tratamento. Atenção especial deve ser dada para a realização do diagnóstico oportuno da infecção pelo HIV em indivíduos com LV, especialmente em homens jovens que residem na zona urbana.

Palavras-chave: Leishmaniose visceral; HIV; Coinfecção; Métodos epidemiológicos.

\begin{abstract}
Introduction: Visceral leishmaniasis (VL) and human immunodeficiency virus (HIV) infection are public health problems, with high numbers of cases and fatality worldwide. Objective: To assess factors associated with leishmania/HIV co-infection in Brazil. Methodology: Retrospective cohort study with univariate and multivariate analysis of 28,265 individuals, notified and with confirmed diagnosis of VL, in the National System of Notifiable Diseases (SINAN), from 2007 to 2018, in Brazil. Results: Most of the co-infected individuals were young, male, nonwhite, living in urban areas, with up to eight years of schooling, and who entered this study as a new case. Greater chances of coinfection were observed for individuals aged from eleven to nineteen years (OR=1.74), twenty to thirty-
\end{abstract}


nine years $(\mathrm{OR}=13.06)$ and over forty years $(\mathrm{OR}=6.96)$, who used $\mathrm{N}$-methylglucamine antimoniate $(\mathrm{OR}=4.36)$, amphotericin $\mathrm{B}$ deoxycholate $(\mathrm{OR}=6.21)$ and liposomal amphotericin $\mathrm{B}(\mathrm{OR}=1.60)$, who had recurrence $(\mathrm{OR}=3.99)$, whose cases evolved to treatment dropout $(\mathrm{OR}=2.00)$, and deaths from causes other than VL $(\mathrm{OR}=4.00)$ and transfer $(\mathrm{OR}=1.50)$. Lower chances of co-infection were observed in women $(\mathrm{OR}=0.80)$, living in rural areas $(\mathrm{OR}=0.54)$, who had five to six symptoms $(\mathrm{OR}=0.82)$. Conclusion: The results point to the need for improvement in the follow-up of individuals co-infected with VL/HIV, in order to reduce relapses, transfers and treatment abandonment. Special attention should be given to the timely diagnosis of HIV infection in individuals with VL, especially in young men living in urban areas.

Keywords: Visceral Leishmaniasis; HIV; Coinfection; Epidemiological methods.

\section{Resumen}

Introducción: La leishmaniasis visceral (LV) y la infección por el virus de la inmunodeficiencia humana (VIH) son problemas de salud pública, con un elevado número de casos y mortalidad en todo el mundo. Objetivo: Evaluar los factores asociados a la coinfección por leishmania / VIH en Brasil. Metodología: Estudio de cohorte retrospectivo con análisis univariado y multivariado de 28.265 individuos, notificados y con diagnóstico confirmado de LV, en el Sistema Nacional de Enfermedades Notificables (SINAN), de 2007 a 2018, en Brasil. Resultados: La mayoría de los coinfectados eran jóvenes, varones, no blancos, residentes en zonas urbanas, con hasta ocho años de escolaridad, y que ingresaron a este estudio como un caso nuevo. Se observaron mayores posibilidades de coinfección en personas de once a diecinueve años $(\mathrm{OR}=1,74)$, de veinte a treinta y nueve años $(\mathrm{OR}=13,06)$ y mayores de cuarenta años $(\mathrm{OR}=6,96)$, que usaban antimoniato de $\mathrm{N}$-metilglucamina $(\mathrm{OR}=4,36)$, desoxicolato de anfotericina $\mathrm{B}(\mathrm{OR}=6,21) \mathrm{y}$ anfotericina $\mathrm{B}$ liposomal $(\mathrm{OR}=1,60)$, que presentaron recidiva $(\mathrm{OR}=3,99)$, cuyos casos evolucionaron a abandono del tratamiento $(\mathrm{OR}=2,00)$, y muertes por causas distintas a la LV ( OR $=4,00)$ y transferencia $(\mathrm{OR}=1,50)$. Se observaron menores probabilidades de coinfección en mujeres $(\mathrm{OR}=0,80)$, que vivían en áreas rurales $(\mathrm{OR}=0,54)$, que tenían de cinco a seis síntomas $(\mathrm{OR}=0,82)$. Conclusión: Los resultados apuntan a la necesidad de mejorar el seguimiento de las personas coinfectadas con LV / VIH, con el fin de reducir las recurrencias, los traslados y el abandono del tratamiento. Se debe prestar especial atención al diagnóstico oportuno de la infección por VIH en personas con LV, especialmente en hombres jóvenes que viven en áreas urbanas.

Palabras clave: Leishmaniasis visceral; VIH; Coinfección; Metodos epidemiologicos.

\section{Introdução}

A Leishmaniose Visceral (LV) é uma antropozoonose generalizada, de evolução crônica e sistêmica, que apesar de ser passível de tratamento e cura, possui elevadas taxas de incidência e letalidade (Leite et al., 2021; Silva et al., 2021). É caracterizada clinicamente por febre, emagrecimento, esplenomegalia e hepatomegalia, e que, quando não tratada, pode evoluir para óbito em mais de $90 \%$ dos casos (Sousa-Gomes et al., 2011).

Apesar dos recursos de tratamento intensivo e das rotinas estabelecidas para o tratamento específico da LV, constatouse, nos últimos anos, aumento na letalidade da doença em diversas regiões do Brasil. Um dos principais fatores que contribuiu para o aumento dessa letalidade é o diagnóstico tardio (Leite et al., 2013). Observou-se que a letalidade da LV no Brasil aumentou, passando de 3,6\% em 1994 para 6,7\% em 2003 e para 8,4 em 2004 (Alvarenga et al., 2010). Na região de Picos, Piauí, nordeste do Brasil, foi encontrada letalidade de 14,1\%, no período de 2007 a 2019 (Abreu et al., 2021). Ressalta-se que o nordeste do Brasil é a região que apresenta maior porcentagem de notificação de LV (Nunes et al., 2020).

O número de casos de coinfecção LV/HIV é crescente em várias partes do mundo, inclusive no Brasil, onde a distribuição geográfica das duas infecções é endêmica (Cota et al., 2012). Leite et al. (2021) encontraram 10\% de coinfectados LV/HIV no Brasil em seu estudo realizado entre os anos de 2007 e 2017, enquanto Costa et al. (2021) encontraram 16,09\% de 2010 a 2019. Essa coinfecção é caracterizada por menor taxa de cura e maior taxa de mortalidade que a monoinfecção por LV. Foi encontrada letalidade três vezes maior em coinfectados com LV/HIV que naqueles sem a coinfecção (Sousa-Gomes et al. 2011). Nos indivíduos coinfectados o número de recidivas é maior e a resposta ao tratamento de LV é menor (Lindoso et al., 2018). Foi descrita associação entre sexo masculino, idade avançada, cor da pele preta e parda e baixa escolaridade com a coinfecção LV/HIV (Couto et al. 2021).

Foram encontrados poucos estudos na literatura avaliando os fatores associados à coinfecção LV/HIV no Brasil, portanto, pretende-se com esta coorte, avaliar a coinfecção LV/HIV no Brasil e os seus fatores associados com intuito de 
fornecer subsídios que poderão ser utilizados na melhoria das ações profiláticas, terapêuticas e no planejamento de intervenções relacionadas a essa coinfecção.

\section{Metodologia}

Este estudo integra o Projeto ECOART (Efetividade da Terapia Antirretroviral em Pessoas Vivendo com HIV, HIV/tuberculose, HIV/hanseníase ou HIV/leishmaniose visceral no Brasil), que é um estudo de coorte prospectivo e retrospectivo no qual são avaliadas pessoas vivendo com HIV (PVHIV) em uso de terapia antirretroviral (TARV) no Brasil. Esse estudo foi aprovado pelo Comitê de Ética em Pesquisa da Universidade Federal de Minas Gerais sob o número de Certificado de Apresentação de Apreciação Ética (CAAE) de 31192914.3.0000.5149, sendo conduzido de acordo com a Resolução 466/2012 do Conselho Nacional de Saúde.

O presente estudo é uma coorte retrospectiva que consiste na análise de dados de todos os indivíduos notificados com LV no Sistema Nacional de Notificação (SINAN), no período de janeiro de 2007 a dezembro de 2018, com diagnóstico laboratorial (parasitológico e/ou molecular) de LV confirmado, residentes no território brasileiro, de todas as idades inclusive bebês, crianças e idosos, com respostas para as variáveis "evolução" ( óbito por LV, óbito por outras causas, cura, transferências e abandono) "infecção por HIV" (sim e não).

O banco do SINAN com dados nominais dos indivíduos foi disponibilizado pela Secretaria de Vigilância em Saúde do Ministério da Saúde. Todos os indivíduos receberam um código de identificação, sendo, posteriormente, anonimizados e desidentificados para a análise. Foram excluídas as múltiplas entradas, isto é, registros no banco com o mesmo nome do indivíduo, da mãe e da data de nascimento.

A variável de medida de resultado foi ter a coinfecção LV/HIV. Essa informação foi obtida no banco de dados de indivíduos notificados e confirmados com LV, de 2007 a 2018, no SINAN, Brasil, por meio da resposta "sim ou não" à pergunta "ter infecção por HIV" no questionário de notificação.

As covariáveis descritas a seguir também foram obtidas do SINAN no qual constavam os seguintes campos: (i) informações relacionadas ao indivíduo e (ii) informações clínicas e relacionadas ao tratamento farmacológico, conforme descritas nos dicionários de dados - (Ministério da Saúde, SINAN). Para interpretação do banco foram consultados dois dicionários do Ministério da Saúde (Brasil, 2018).

Variáveis relacionadas ao indivíduo: sociodemográficas: data de nascimento, idade na data de notificação, sexo, raça, gestante, escolaridade, estado de moradia, zona de moradia e ocupação. Variáveis clínicas: data da notificação, tipo de diagnóstico, número e tipos de sintomas, data do óbito, classificação final, critério de confirmação, data de encerramento, tipo de entrada (caso novo, recidiva e transferência) e evolução (óbito, cura, abandono e transferência). Variáveis relacionadas ao tratamento farmacológico: uso e tipos de medicamentos iniciais administrados.

As análises estatísticas foram todas realizadas no software Stata 12.0 com chave de licença EYGZ-H2J4-M5N6Q8R9-SBUC-VDXF.

Foi realizada a análise descritiva por distribuição de frequências, absoluta e relativa, para variáveis estratificadas pelas categorias do desfecho, considerando um intervalo de confiança de 95\% (IC 95\%). Para analisar a associação entre as variáveis foi utilizado o Teste Qui-Quadrado de Pearson. Na análise univariada e multivariada utilizou-se o modelo de regressão logística binomial. Consideramos para o modelo multivariado todas as variáveis que apresentaram valor "p" inferior a 0,20 na análise univariada. Partiu-se do modelo completo com descarte sucessivo das variáveis que ajustadas em relação às demais (método de backward) não apresentaram nível de significância de $p<0,05$. Permaneceram no modelo logístico binomial final as variáveis que apresentaram nível de significância estatística $(\mathrm{p}<0,05)$. Para definição dos modelos finais, foi utilizado o teste da razão da verossimilhança (Hosmer \& Lemeshow, 1989). 


\section{Resultados e Discussão}

A população de estudo foi selecionada do banco de dados que inicialmente apresentava 113.495 indivíduos. Foram excluídos 3.475 registros múltiplos, restando 110.020 registros únicos (96,93\%). Dos registros únicos foram excluídos 66.657 registros de indivíduos que não apresentaram confirmação diagnóstica de LV, sendo selecionados, 43.363 indivíduos com diagnóstico confirmados $(38,21 \%)$. Dentre esses, foram excluídos 5.780 registros de indivíduos que não apresentaram resposta para a variável “evolução” no SINAN. Dos 37.583 indivíduos $(33,11 \%)$ foram excluídos 9.318 que não responderam a variável infecção por HIV. O número de registros analisados foi 28.265 (25,69\%) do total de indivíduos notificados entre 2007 a 2018 , no SINAN, Brasil (Figura 1).

Figura 1. Total de casos notificados entre 2007 a 2018, no SINAN, Brasil.

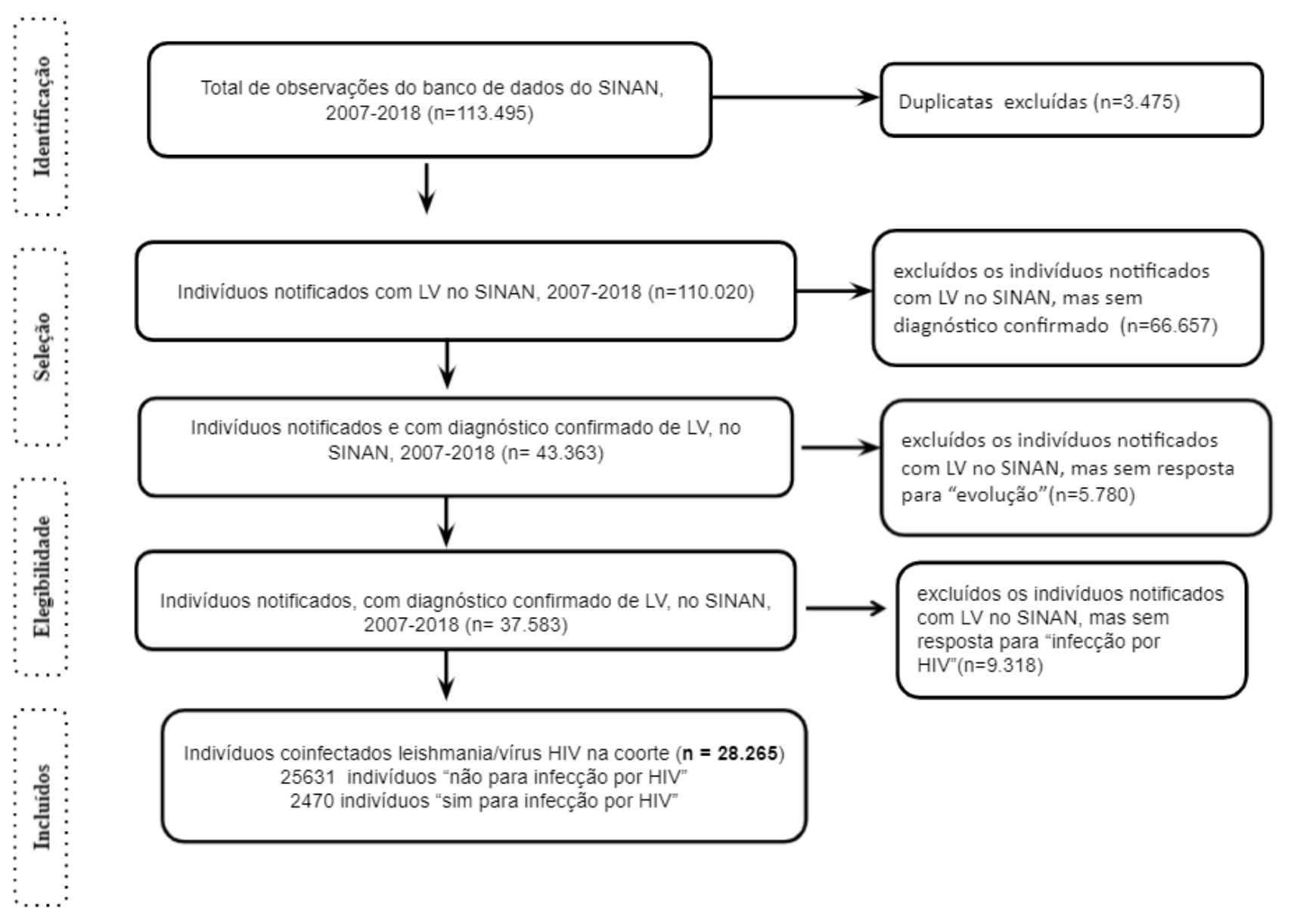

Observação: 164 indivíduos são dados faltantes. Fonte: Autores (2021).

Dentre os 28.265 indivíduos da coorte que apresentaram resposta para a variável infecção por HIV, $2.489(8,81 \%)$ indivíduos relataram ter infecção por HIV. O maior percentual de indivíduos coinfectados estava na faixa etária entre 20 a 39 anos (49,3\%), a maioria era do sexo masculino (77,6\%), raça não branca (83,5\%), com até oito anos de escolaridade (75,6\%), moradores da zona urbana $(87,3 \%)$. Houve um percentual elevado de indivíduos que apresentaram de sete a doze sintomas $(45,5 \%)$. Dentre os sintomas, os mais relatados foram febre $(87,3 \%)$, fraqueza $(84,6 \%)$, emagrecimento $(81,5 \%)$, aumento do baço $(72,6 \%)$, palidez $(72,5 \%)$ e aumento do fígado (66,5\%). Quanto ao tipo de entrada no banco do SINAN, a maioria $(89,0 \%)$ foi de casos novos. Em relação ao tratamento da LV, 69,4\% utilizaram Anfotericina B e 24,6\% utilizaram Antimoniato De N-Metilglucamina. A maioria (72,1\%) evoluíram para cura. Quanto ao ano de notificação, observou-se que a proporção de indivíduos coinfectados era de 4,0\% em 2007, aumentando gradativamente até 10,1\% em 2012, com redução em 
2013 (7,9\%), $2014(8,6)$ e 2015 (8,5\%). Houve maior aumento de notificação nos anos de 2016 e 2017 (11,1\% e 11,5\% respectivamente). Quanto às regiões, a região nordeste apresentou o maior

Tabela 1. Análise descritiva dos indivíduos notificados e com diagnóstico confirmado de LV, no SINAN, estratificado segundo a infecção por HIV, no período de 2007 a 2018, Brasil (n=28.265).

\begin{tabular}{|c|c|c|c|c|c|c|}
\hline \multirow{3}{*}{ Variável } & \multicolumn{4}{|c|}{ HIV } & \multirow{2}{*}{\multicolumn{2}{|c|}{ Total }} \\
\hline & \multicolumn{2}{|c|}{ Negativo } & \multicolumn{2}{|c|}{ Positivo } & & \\
\hline & $\mathrm{n}$ & $\%$ & $\mathrm{n}$ & $\%$ & \multirow[t]{2}{*}{$\mathrm{n}$} & \multirow[t]{2}{*}{$\%$} \\
\hline Idade (anos) & & & & & & \\
\hline 0 a 4 & 10.998 & 37,5 & 143 & 5,1 & 11.141 & 34,7 \\
\hline 5 a 19 & 5.937 & 20,2 & 138 & 4,9 & 6.075 & 18,9 \\
\hline 20 a 39 & 5.949 & 20,3 & 1.381 & 49,3 & 7.330 & 22,8 \\
\hline 40 a 59 & 4.248 & 14,5 & 1.004 & 35,9 & 5.252 & 16,3 \\
\hline $60+$ & 2.195 & 7,5 & 133 & 4,8 & 2.328 & 7,2 \\
\hline \multicolumn{7}{|l|}{ Sexo } \\
\hline Masculino & 18.534 & 63,2 & 2.171 & 77,6 & 20.705 & 64,5 \\
\hline Feminino & 10.792 & 36,8 & 627 & 22,4 & 11.419 & 35,5 \\
\hline \multicolumn{7}{|l|}{ Raça } \\
\hline Branca & 4.369 & 15,9 & 427 & 16,5 & 4.796 & 16,0 \\
\hline Não branca & 23.093 & 84,1 & 2.155 & 83,5 & 25.248 & 84,0 \\
\hline \multicolumn{7}{|l|}{ Escolaridade (anos) } \\
\hline Até 8 & 8.933 & 78,6 & 1.200 & 75,6 & 10.133 & 78,2 \\
\hline 9 a 12 & 2.184 & 19,2 & 323 & 20,3 & 2.507 & 19,3 \\
\hline 12 ou mais & 252 & 2,2 & 65 & 4,1 & 317 & 2,4 \\
\hline \multicolumn{7}{|l|}{ Zona } \\
\hline Urbana & 20.926 & 73,5 & 2.358 & 87,3 & 23.284 & 74,7 \\
\hline Rural & 7.242 & 25,4 & 321 & 11,9 & 7.563 & 24,3 \\
\hline Periurbana & 289 & 1,0 & 23 & 0,9 & 312 & 1,0 \\
\hline \multicolumn{7}{|l|}{ Tipos de Sintoma } \\
\hline Febre & 27.600 & 94,4 & 2.409 & 87,3 & 30.009 & 93,8 \\
\hline Fraqueza & 23.510 & 81,2 & 2.318 & 84,6 & 25.828 & 81,5 \\
\hline Edema & 7.244 & 25,6 & 606 & 23,0 & 7.850 & 25,4 \\
\hline Emagrecimento & 20.536 & 71,1 & 2.236 & 81,5 & 22.772 & 72,0 \\
\hline Tosse e/ou diarreia & 12.563 & 43,7 & 1.493 & 55,3 & 14.056 & 44,7 \\
\hline Palidez & 21.304 & 74,5 & 1.947 & 72,5 & 23.251 & 74,3 \\
\hline Aumento do baço & 23.044 & 80,2 & 1.949 & 72,6 & 24.993 & 79,6 \\
\hline Quadro Infeccioso & 6.637 & 23,9 & 945 & 36,5 & 7.582 & 25,0 \\
\hline Fenômeno hemorrágico & 2.838 & 10,1 & 392 & 15,1 & 3.230 & 10,5 \\
\hline Aumento do fígado & 19.735 & 69,2 & 1.784 & 66,5 & 21.519 & 68,9 \\
\hline Icterícia & 7.004 & 24,8 & 474 & 18,2 & 7.478 & 24,2 \\
\hline Outros & 6.017 & 22,2 & 716 & 28,4 & 6.733 & 22,7 \\
\hline \multicolumn{7}{|l|}{ Sintomas } \\
\hline 0 a 4 & 6.381 & 21,8 & 654 & 23,4 & 7.035 & 21,9 \\
\hline 5 a 6 & 10.716 & 36,5 & 871 & 31,1 & 11.587 & 36,1 \\
\hline 7 a 12 & 12.230 & 41,7 & 1.274 & 45,5 & 13.504 & 42,0 \\
\hline \multicolumn{7}{|l|}{ Entrada } \\
\hline Novo & 27.944 & 96,4 & 2.459 & 89,0 & 30.403 & 95,7 \\
\hline Recidiva & 636 & 2,2 & 269 & 9,7 & 905 & 2,8 \\
\hline
\end{tabular}


Research, Society and Development, v. 10, n. 17, e03101724545, 2021

(CC BY 4.0) | ISSN 2525-3409 | DOI: http://dx.doi.org/10.33448/rsd-v10i17.24545

\begin{tabular}{|c|c|c|c|c|c|c|}
\hline Transferência & 419 & 1,4 & 34 & 1,2 & 453 & 1,4 \\
\hline \multicolumn{7}{|l|}{ Medicamento } \\
\hline Não & 878 & 3,2 & 97 & 3,7 & 975 & 3,2 \\
\hline Antimoniato Pentavalente & 18.118 & 65,2 & 653 & 24,6 & 18.771 & 61,6 \\
\hline Anfotericina & 7.878 & 28,3 & 1.839 & 69,4 & 9.717 & 31,9 \\
\hline Outras & 926 & 3,3 & 61 & 2,3 & 987 & 3,2 \\
\hline \multicolumn{7}{|l|}{ Evolução } \\
\hline Cura & 21.366 & 82,9 & 1.795 & 72,1 & 23.161 & 81,9 \\
\hline Abandono & 191 & 0,7 & 32 & 1,3 & 223 & 0,8 \\
\hline Óbito LV & 1.892 & 7,3 & 274 & 11,0 & 2.166 & 7,7 \\
\hline Óbito Outras Causa & 451 & 1,7 & 248 & 10,0 & 699 & 2,5 \\
\hline Transferência & 1.876 & 7,3 & 140 & 5,6 & 2.016 & 7,1 \\
\hline \multicolumn{7}{|l|}{ Ano de notificação } \\
\hline 2007 & 2.453 & 8.4 & 112 & 4.0 & 2,565 & 8.0 \\
\hline 2008 & 2.473 & 8.4 & 156 & 5.6 & 2,629 & 8.2 \\
\hline 2009 & 2.548 & 8.7 & 211 & 7.5 & 2,759 & 8.6 \\
\hline 2010 & 2.387 & 8.1 & 206 & 7.4 & 2,593 & 8.1 \\
\hline 2011 & 2.564 & 8.7 & 233 & 8.3 & 2,797 & 8.7 \\
\hline 2012 & 2.069 & 7.1 & 284 & 10.1 & 2,353 & 7.3 \\
\hline 2013 & 2.268 & 7.7 & 220 & 7.9 & 2,488 & 7.7 \\
\hline 2014 & 2.350 & 8.0 & 241 & 8.6 & 2,591 & 8.1 \\
\hline 2015 & 2.262 & 7.7 & 241 & 8.6 & 2,503 & 7.8 \\
\hline 2016 & 2.214 & 7.5 & 312 & 11.1 & 2,526 & 7.9 \\
\hline 2017 & 3.050 & 10.4 & 321 & 11.5 & 3,371 & 10.5 \\
\hline 2018 & 2.689 & 9.2 & 262 & 9.4 & 2,951 & 9.2 \\
\hline \multicolumn{7}{|l|}{ Mês de notificação } \\
\hline Janeiro & 2.594 & 8.8 & 259 & 9.3 & 2,853 & 8.9 \\
\hline Fevereiro & 2.172 & 7.4 & 219 & 7.8 & 2,391 & 7.4 \\
\hline Março & 2.439 & 8.3 & 241 & 8.6 & 2,68 & 8.3 \\
\hline Abril & 2.349 & 8.0 & 212 & 7.6 & 2,561 & 8.0 \\
\hline Maio & 2.528 & 8.6 & 233 & 8.3 & 2,761 & 8.6 \\
\hline Junho & 2.517 & 8.6 & 238 & 8.5 & 2,755 & 8.6 \\
\hline Julho & 2.662 & 9.1 & 247 & 8.8 & 2,909 & 9.1 \\
\hline Agosto & 2.764 & 9.4 & 261 & 9.3 & 3,025 & 9.4 \\
\hline Setembro & 2.558 & 8.7 & 249 & 8.9 & 2,807 & 8.7 \\
\hline Outubro & 2.441 & 8.3 & 233 & 8.3 & 2,674 & 8.3 \\
\hline Novembro & 2.226 & 7.6 & 217 & 7.8 & 2,443 & 7.6 \\
\hline Dezembro & 2.077 & 7.1 & 190 & 6.8 & 2,267 & 7.1 \\
\hline \multicolumn{7}{|l|}{ Região geográfica } \\
\hline Norte & 5.314 & 18.1 & 205 & 7.3 & 5,519 & 17.2 \\
\hline Nordeste & 15.790 & 53.9 & 1.530 & 54.8 & 17,33 & 54.0 \\
\hline Sudeste & 5.803 & 19.8 & 662 & 23.7 & 6,465 & 20.1 \\
\hline Sul & 78 & 0.3 & 8 & 0.3 & 86 & 0.3 \\
\hline Centro Oeste & 2.326 & 7.9 & 388 & 13.9 & 2,714 & 8.5 \\
\hline
\end{tabular}

* valores variam devido a dados faltantes. Fonte: Autores (2021). 
$\mathrm{Na}$ análise univariada (Tabela 2) foram observadas maiores chances de coinfecção LV/HIV para indivíduos na faixa etária entre 20 a 39 anos $(\mathrm{OR}=15,64)$, sexo feminino $(\mathrm{OR}=1,05)$, com escolaridade acima de 12 anos de estudo (OR=1,83), que utilizaram anfotericina $\mathrm{B}$ desoxicolato $(\mathrm{OR}=7,8)$, que apresentaram recidiva $(\mathrm{OR}=4,73)$, que evoluíram para óbito por outras causas que não LV $(\mathrm{OR}=6,18)$. Observaram-se, ainda, menores chances da coinfecção LV/HIV para os indivíduos moradores das zonas rural $(\mathrm{OR}=0,4)$, para aqueles que apresentaram de cinco a seis sintomas $(\mathrm{OR}=0,80)$.

$\mathrm{Na}$ análise multivariada (Tabela 2), os fatores significativamente associados a maiores chances de coinfecção LV/HIV foram faixa etária acima de 10 anos ( 11 a 19 anos $\mathrm{OR}=1,74$; entre 20 a 39 anos $\mathrm{OR}=13,06$ e acima de 40 anos $\mathrm{OR}=6,96$ ), uso de medicamentos antimoniato pentavalente, anfotericina B desoxicolato, anfotericina B lipossomal e outros medicamentos $(\mathrm{OR}=4,36 ; 6,21 ; 1,60$ e 1,73 respectivamente), que tiveram entrada como recidiva $(\mathrm{OR}=3,99)$ e que evoluíram para abandono do tratamento, óbito por outras causas que não LV ou transferência ( $\mathrm{OR}=2,00 ; 4,00$ e 1,50 respectivamente).

Os fatores significativamente associados a menores chances de coinfecção LV/HIV foram ser do sexo feminino $(\mathrm{OR}=0,80)$ e residir em zona rural $(\mathrm{OR}=0,54)$.

Tabela 2. Análise univariada e multivariada dos fatores associados a coinfecção LV/HIV em indivíduos notificados e com diagnóstico confirmado de LV, no SINAN, no período de 2007 a 2018, Brasil (n=28.265).

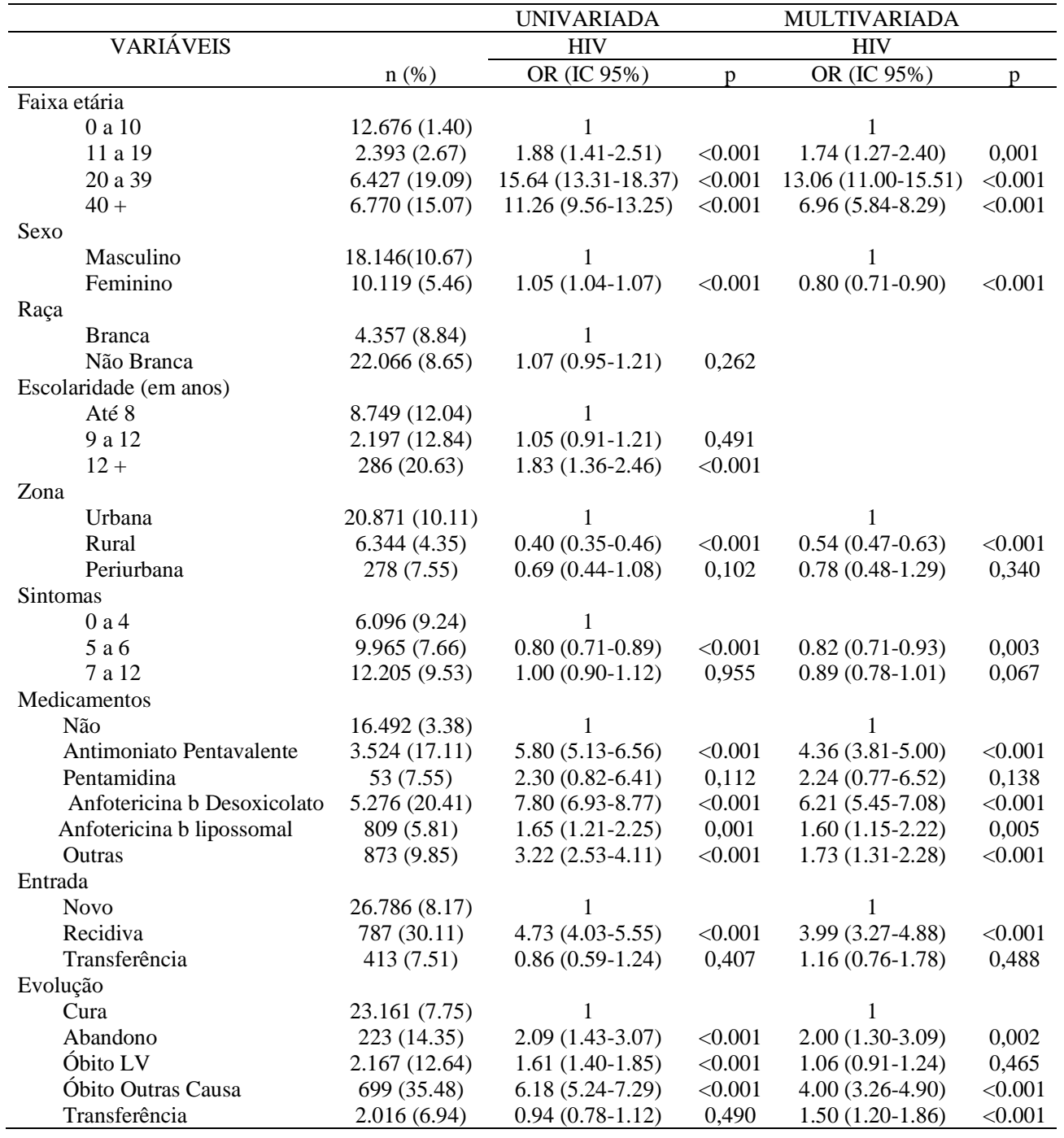

Fonte: Autores (2021).

A maioria dos indivíduos coinfectados era homem, jovem, da raça não branca, moradores da zona urbana, com até 
oito anos de escolaridade e que apresentavam caso novo de infecção por LV. Os fatores significativamente associados a maiores chances de coinfecção LV/HIV foram faixa etária acima de 10 anos, uso de anfotericina, apresentaram recidiva de infecção por LV e que evoluíram para abandono do tratamento, óbito por outras causas que não LV ou transferência. Os fatores significativamente associados a menores chances de coinfecção LV/HIV foram ser do sexo feminino e residir em zona rural.

Indivíduos da raça/cor de pele não brancas foram a maioria dentre os coinfectados no estudo, semelhante ao estudo de Costa et. al. (2021) desenvolvido em São Luís do Maranhão, no qual a maioria dos indivíduos coinfectados com LV/HIV, entre os anos de 2010 a 2019, eram da raça/cor de pele preta. Oliveira et.al. (2021) descreveram predominância da raça parda entre os indivíduos notificados por LV no Brasil.

Indivíduos com faixas etárias maiores que 11 anos apresentaram maiores chances de serem coinfectados com LV/HIV, no estudo. Estes dados estão consonantes com outro estudo onde foi observado que o aumento da idade, o decréscimo da resposta terapêutica, e a presença de comorbidades são fatores associados à reincidência de infecções oportunistas como LV, em indivíduos diagnosticados com HIV (Dembelu \& Woseneleh, 2021). Ademais, Alvarenga et. al. (2010) observaram que indivíduos com maior idade apresentaram maior percentual de comorbidades e que a maior sobrevivência foi observada nos indivíduos que não possuíam comorbidades associadas à LV.

No presente estudo os indivíduos que utilizaram anfotericina B apresentaram a maior chance de serem coinfectados com LV/HIV, em consonância com as recomendações do Ministério da Saúde, estabelecidas a partir de 2009, que passou a recomendar a utilização de anfotericina B como medicamento de escolha para indivíduos coinfectados com LV/HIV (Brasil, 2009). Souza-Gomes et al. (2011) observaram um aumento no uso de anfotericina B em relação ao então medicamento de escolha, antimoniato de n-metilglucamina, já no ano de 2008, quando esse uso foi comparado com o do ano de 2007, no Brasil. Dembelu e Woseneleh (2021) relataram que a adesão ao tratamento antirretroviral foi fator associado à reincidência de infecções oportunistas, como LV, em indivíduos diagnosticados com HIV.

Indivíduos com recidiva de infecção por LV apresentaram maiores chances de terem a coinfecção LV/HIV, no estudo, resultado semelhante ao de Silva et al. (2021) que observou presença de recidiva duas vezes maior em indivíduos coinfectados com LV/HIV. As recidivas de infecção por LV foram associadas à alta letalidade em indivíduos coinfectados por SousaGomes et al. (2017). Portanto maior atenção precisa ser dada ao acompanhamento dos indivíduos com LV com relação ao diagnóstico oportuno de HIV.

Os indivíduos que evoluíram para abandono do tratamento, óbito por outras causas que não LV ou transferência foram significativamente associados a maiores chances de coinfecção LV/HIV. A dificuldade dos indivíduos de aceitarem o diagnóstico da infecção por HIV pode relacionar-se com abandono de tratamento, óbitos por abandono e transferência de serviço de saúde (Gomes et al., 2021).

No presente estudo a chance de indivíduos do sexo feminino serem coinfectados com LV/HIV foi menor. Provavelmente, devido a maior incidência de HIV no sexo masculino. A infecção por HIV iniciou-se, na década de 80, em indivíduos do sexo masculino, por via sexual desprotegida (Batista et al., 2021) e mantém até o período do estudo maior número de notificações para indivíduos do sexo masculino. Ademais os homens apresentam atividades comportamentais de maior risco o que os tornam mais susceptíveis à infecção por HIV (Mazon-Silva et al., 2021).

Indivíduos que residem em zonas rurais apresentaram menores chances de terem a coinfecção LV/HIV, estando de acordo com os estudos de Carvalho et al. (2013) e Silva et al. (2021) que reportaram em seus estudos que os indivíduos infectados com HIV, sem ou com LV, eram procedentes da área urbana. Entretanto, Monteiro et al. (2021), afirmaram que está infecção tem expandido ao longo dos últimos anos para as periferias das grandes cidades, pequenas cidades e zona rural, com permanência no meio urbano. 
No presente estudo os indivíduos com cinco a seis sintomas de LV apresentaram menores chances de coinfecção, o que pode ser decorrente da utilização de banco de dados de notificação de indivíduos com LV. Os principais sintomas de LV notificados são febre, perda de peso, anemia e esplenomegalia conforme revisão integrativa de literatura (Souza et. al. 2020).

Como limitações, os dados deste estudo foram obtidos de banco administrativo do Ministério da Saúde e, portanto, o banco não foi desenvolvido para pesquisa científica, não sendo possível a coleta de informações clínicas relacionadas aos sintomas e à severidade da infecção, bem como os motivos das transferências dos indivíduos entre unidades de saúde. Observou-se, também, incompletude de dados para algumas variáveis. Como pontos fortes do trabalho relata-se a robustez das análises estatísticas realizadas, abrangência do banco por ser nacional e o extenso período de acompanhamento dos indivíduos.

\section{Conclusão}

Os fatores independentemente associados e que aumentam a chance dos indivíduos com LV terem infecção por HIV, quais sejam entradas por recidiva e que evoluíram para abandono do tratamento, óbitos por outras causas que não LV e transferência, podem sugerir a necessidade de melhorias na qualidade do acompanhamento desses indivíduos nos serviços de saúde. Necessário se faz também, buscar a vinculação dos indivíduos ao tratamento e ao serviço de saúde em consonância com os princípios de universalidade, integralidade e equidade do Sistema Único de Saúde. Atenção especial deve ser dada para a realização do diagnóstico oportuno da infecção pelo HIV em indivíduos com LV, especialmente em homens jovens que residem na zona urbana.

Para trabalhos futuros, tem-se como sugestão realizar o estudo da efetividade do tratamento em indivíduos coinfectados com Leishmania/vírus da imunodeficiência humana, por meio da associação entre o banco de dados desse estudo com o banco de dados do Sistema de Controle de Logística de Medicamentos (SICLOM).

\section{Agradecimentos}

Ao grupo técnico de leishmanioses da Secretaria de Vigilância em Saúde do Ministério da Saúde pela disponibilidade do banco de dados nominais da leishmaniose visceral referente ao período de 2007 a 2018 . À Marcia Sousa Gomes pela presteza em esclarecer as dúvidas acerca do banco de dados.

\section{Referências}

Abreu, M. S., Siqueira, J. M. M. T., Maia, J. C. S., Nepomuceno, D. B., Luz, E. B. A. L., \& Mendes-Sousa, A. F. (2021). Aspectos epidemiológicos e distribuição espacial da leishmaniose visceral em Picos, Piauí, Brasil. Saúde Coletiva (Barueri), $11(65)$, 5846-5857. https://doi.org/10.36489/saudecoletiva.2021vl1i65p5 846-5857

Alvarenga, D. G., Escalda, P. M. F., Costa, A. S. V., \& Monreal, M. T. F. D. (2010). Leishmaniose visceral: estudo retrospectivo de fatores associados à letalidade. Revista da Sociedade Brasileira de Medicina Tropical, 43(2), 194-197. https://doi.org/10.1590/S0037-86822010000200017

Batista, R. M., Andrade, S. S., \& Souza, T. F. M. P. (2021). Prevalence of HIV / AIDS cases in the last 10 years in Brazil. Research, Society and Development, 10(14), 01-08 https://doi.org/10.33448/rsd-v10i14.22149

Brasil. (2009) Ministério da Saúde. Guia de Vigilância Epidemiológica. (7a ed.), Ministério da Saúde.

Brasil. (2012). Ministério da Saúde. Resolução n 466, de 12 de dezembro de 2012. Aprova diretrizes e normas regulamentadoras de pesquisas envolvendo seres humanos. Diário Oficial da União, 12(1), 59-63. https://conselho.saude.gov.br/resolucoes/2012/Reso466.pdf

Brasil. (2018). Ministério da Saúde. SINAN - Sistema de Agravo de Notificação. Ministério da Saúde, Brasília. http://sinan.saude.gov.br/sinan/login/login.jsf

Carvalho, F. L., Aires, D. L. S., Segunda, Z. F., Azevedo, C. M. P. S., Corrêa, R. G. C. F., Aquino, D. M. C., \& Caldas, A. J. M. (2013). Perfil epidemiológico dos indivíduos HIV positivo e coinfecção HIV-Leishmania em um serviço de referência em São Luís, MA, Brasil. Ciência \& Saúde Coletiva, 18(5), 13051312. https://doi.org/10.1590/S1413-81232013000500015.

Costa, R. K. E., Holanda, E. C., Andrade, S. M., Nascimento, M. S. V., Soares, L. F., \& Oliveira, E. H. (2021). Visceral Leishmaniasis and Human Immunodeficiency Virus Coinfection: epidemiological profile of cases reported in São Luís-Maranhão, Brazil. Research, Society and Development, 10(4), 0110. https://doi.org/1 0.33448/rsd-v10i4.13317. 
Cota, G. F., Sousa, M. R., Demarqui, F. N., \& Rabello, A. (2012). The diagnostic accuracy of serologic and molecular methods for detecting visceral leishmaniasis in HIV infected patients: meta-analysis. PLoS Neglectd Tropical Diseases, 6(5), 01-11. https://doi.org/10.1371/journal.pntd.0001665.

Couto, F. M., Santos, A. B., Domiciano, L. S., Reis, L. E. S. (2021). Vínculo fatal -Leishmania infantum e HIV: uma revisão sistemática de literatura. Revista da Semana Acadêmica do Curso de Medicina da UFFS, 4(4), 01-13.

Dembelu, M., \& Woseneleh, T. (2021). Prevalence of and Factors Associated with Reoccurrence of Opportunistic Infections Among Adult HIV/AIDS Patients Attending the ART Clinic at Public Health Facilities in Arba Minch Town, Southern Ethiopia. HIV/AIDS (Auckland, N.Z.), 13(1), 867-876. https://doi.org/10.2147/HIV.S328362

Gomes MP, Barbosa D, Gomes AMT, Silva G, Souza FA, \& Silva ALB. (2021). A vivência do preconceito após a revelação da soropositividadae para HIV. Revista Rede de Cuidados em Saúde, 15(1), 47-56.

Hosmer, D. W., Jovanovic, B., \& Lemeshow, S. (1989). Best Subsets Logistic Regression. Biometrics, 45(4), 1265-1270. https://doi.org/10.2307/2531779

Leite, A. I., \& Araújo, L. B. (2013). Leishmaniose visceral: aspectos epidemiológicos relacionados aos óbitos em Mossoró-RN. Revista de Patologia Tropical, 42(3), 301-308. https://doi.org/10.5216/rpt.v42i3.2 6928

Leite, N. C., Garcia, J. L., \& Gonçalves, I. M. (2021). Perfil epidemiológico da leishmaniose visceral no Brasil no período de 2007 a 2017. Revista de Patologia do Tocantins, 7(4), 29-33. https://doi.org/10.20873/ uft.2446-6492.2020v7n4p29

Lindoso, J. A. L., Moreira, C. H. V., Cunha, M. A.; \& Queiroz, I. T. (2018). Visceral leishmaniasis and HIV coinfection: current perspectives. HIV/AIDS Research and Palliative Care, 10(1), 193-201. https://d oi.org/10.2147/HIV.S143929

Mazon-Silva, S. L., Oliveira, N. A., Aguiar, A. S., Guimarães, N. S., \& Figueiredo, S. M. (2021). Consumo alcoólico de homens soropositivos para o HIV. Health and Biosciences, 2(1), 05-15. https://doi.org/10.4745 6/hb.v2i1.32459

Monteiro, I. O. P., Leite, E. H. C. A. M., Costa, M. S. S., Souza, L. S., Pinto, E. O., Almeida, G. S., \& Cordeiro, P. M. (2021). The Human Immunodeficiency Virus in rural areas: reflections from the perspective of access to health services. Research, Society and Development, 10(11), 01-16. https://doi.org/10.33448/rs d-v10i11.19620

Nunes, B. E. B. R., Leal, T. C., Paiva, J. P. S., Silva, L. F., Carmo, R. F., Machado, M. F., Araújo, M. D. P., Santos, V. S., \& Souza, C. D. F. (2020). Social determinants of mortality due to visceral leishmaniasis in Brazil (2001-2015): an ecological study. Revista da Sociedade Brasileira de Medicina Tropical, 53(1): e20190262. https://doi.org/10.1590/0037-8682-0262-2019

Oliveira, L. C. (2021). Aspectos epidemiológicos da leishmaniose visceral humana no Brasil e estados e cidades gêmeas do país, com enfoque no estado e na cidade gêmea com maior número de casos. [Dissertação de mestrado, Universidade Estadual do Oeste do Paraná]. http://tede.unioeste.br/handle/tede/5 540

Silva, B. B. L., Alves, A. K. R., Almeida, B. C., Pereira, R. B., Castro, M. R., Santos, V. A. P. P., Fontenele, M. J. A., Silva, L. S., Oliveira, A. C., Nogueira, F. D., Alves, F. R. O., Veras, I. L., Silveira, E. M. Q. F. B., Mello, G. W. S., \& Farias, D. R. (2021). Análise dos perfis epidemiológicos da leishmaniose visceral e da coinfecção leishmaniose visceral-HIV no Piauí, 2007 a 2019. Research, Society and Development, 10(12), 01-08. http://dx.doi.org/10.33448/rsdv10i12.20247

Sousa-Gomes, M. L., Maia-Elkhoury, A. N. S., Pelissari, D. M.; Lima Júnior, F. E. F., Sena, J. M., Chechinel, M. P. (2011). Coinfecção Leishmania-HIV no Brasil: aspectos epidemiológicos, clínicos e laboratoriais. Epidemiologia e Serviços de Saúde, 20(4), 519-526.http://dx.doi.org/10.5123/S167949742011000400011

Sousa-Gomes, M. L., Romero, G. A. S., \& Werneck, G. L. (2017). Visceral leishmaniasis and HIV/AIDS in Brazil: Are we aware enough? PLoS Neglected Tropical Diseases, 11(9), 01-12. http://dx.doi.org/10.137 1/journal.pntd.0005772

Souza, E. C., Braga, K. L., Silva, T. K., Silva, M. L. (2020). Apresentação clínica da leishmaniose visceral em pacientes portadores do HIV: Análise dos Fatores Relacionados ao Aparecimento da Doença. Brazilian Journal of health Review, 3(2), 1766-1777. DOI:10.34119/bjhrv3n2-037 